\title{
Bounds for the Adiabatic Approximation with Applications to Quantum Computation
}

\author{
Sabine Jansen* \\ Institut für Mathematik, MA 7-2, TU Berlin \\ Straße des 17. Juni 136 \\ D-10623 Berlin, Germany \\ jansen@math.tu-berlin.de, \\ Mary Beth Ruskai ${ }^{\dagger}$ \\ Department of Mathematics, Tufts University, Medford, MA 02155 \\ Marybeth.Ruskai@tufts.edu \\ Ruedi Seiler $\ddagger$ \\ Institut für Mathematik, MA 7-2, TU Berlin \\ seiler@math.tu-berlin.de \\ 11th May 2018 \\ Dedicated to Barry Simon on the occasion of his 60th birthday
}

\begin{abstract}
We present straightforward proofs of estimates used in the adiabatic approximation. The gap dependence is analyzed explicitly. We apply the result to interpolating Hamiltonians of interest in quantum computing.
\end{abstract}

\footnotetext{
${ }^{*}$ Supported by the Deutsche Forschungsgemeinschaft (DFG)

${ }^{\dagger}$ Partially supported by the National Security Agency (NSA) and Advanced Research and Development Activity (ARDA) under Army Research Office (ARO) contract number DAAD19-02-10065, and by the National Science Foundation under Grant DMS-0314228.

${ }^{\ddagger}$ Supported by the Deutsche Forschungsgemeinschaft (DFG)
} 


\section{Introduction}

The quantum adiabatic approximation has a long history, going back to Born and Fock $[\mathrm{BF}]$ early in the development of quantum theory. Recently, the realization that the adiabatic approximation could be used as the basis for a method of quantum computation [FGGS] has generated a resurgence of interest in this topic.

Despite the existence of an extensive literature ASY1, HJ, K, N1, JP] on rigorous proofs of estimates needed to justify the adiabatic approximation, doubts have been raised about its validity $[\mathrm{MS}$ ] leading to confusion about the precise conditions needed to use it AR, TSKO, MEP. In part, this is because some papers emphasize different aspects, such as the asymptotic expansion, the replacement of the requirement of a non-degenerate ground state by a spectral projection separated from the rest of the spectrum, dependence of first order estimates on the spectral gap, and even extensions to systems without a gap. Moreover, the qualitative gap conditions frequently presented in elementary texts [LL, M] are known to be insufficient.

In this paper we present a straightforward, yet rigorous, proof of the asymptotic estimates in a form which makes explicit the relevance of the gap. The proof given here is based on the one in ASY1 with the modifications introduced in ASY2 and [KS. The main idea is to consider the physical time evolution as a perturbation of the adiabatic time evolution. This leads naturally to an integration by parts formula which is the main technical tool.

Roughly speaking, the adiabatic approximation says that when a Hamiltonian changes slowly in time, the corresponding time-evolution approximately preserves spectral subspaces. In particular an eigenstate $\psi(0)$ evolves with high probability to the eigenstate $\psi(t)$ of the instantaneous Hamiltonian $H(t)$ if the energy curve $E(t)$ does not come too close to any other energy level of $H(t)$. To make this precise, one considers the family of Hamiltonians $H\left(\frac{t}{\tau}\right)$ where $t$ should be thought of as the "external" clock time and the parameter $\tau$ defines a time scale which allows tuning of the speed of change of the Hamiltonian. It gives rise to a scaled time $s=\frac{t}{\tau}$. Using this notation time evolution is defined in terms of the family of time dependent Schrödinger equations

$$
i \partial_{t} \phi_{\tau}(t)=H(t / \tau) \phi_{\tau}(t)
$$

or equivalently

$$
i \partial_{s} \psi_{\tau}(s)=\tau H(s) \psi_{\tau}(s) .
$$

In the most common scenario, the adiabatic theorem is an asymptotic expansion in

$\frac{1}{\tau}$ for the error involved in estimating the time-evolved ground state of $H(0)$ by the ground state of $H(s)$.

Remark: Allowed time-dependence. The adiabatic theorems give results when the time-dependence of the Hamiltonian in the unscaled time $t$ is of the form $H(t / \tau)$ for some $\tau$-independent family of operators $H(\cdot)$. 
It is important to understand that this excludes Hamiltonians with several independent time scales. In particular, the example by Marzlin and Sanders ( involves a Hamiltonian $H\left(t / \tau, \omega_{0} t\right)$ with fixed frequency $\omega_{0}$ and big time scale $\tau$ does not belong to this class. To see this, notice that in terms of the dimensionless time $s=t / \tau$, the Hamiltonian is $H\left(s, \omega_{0} \tau s\right)$ which is $\tau$-dependent unless $\omega_{0} \tau$ is taken to be constant.

In adiabatic quantum computation [FGGS, [FGG1], the Hamiltonians of interest are usually interpolating Hamiltonians of the form

$$
H(s)=(1-f(s)) H_{0}+f(s) H_{1}
$$

Generalizations of the form

$$
H(s)=(1-f(s)) H_{0}+f(s) H_{1}+k(s) H_{2}
$$

with $k(0)=k(1)=0$ have also been considered [FGG2]. In most applications of the adiabatic theorem to quantum computation, there is another parameter, $n$ which describes the size of the system, and one is interested in the behavior of a family of quantum system when $n$ is large. In particular, one is often interested how certain quantities, such as the running time of a computer program, grow (or decrease) with $n$. This brings a new element into the discussion of the adiabatic approximation and requires a careful analysis of the error bound in terms of the gap of a family of quantum systems. We present such estimates in Theorems 3 and 4 of this paper.

The essential assumption in the adiabatic theorem as it is presented here is that the spectrum $\sigma(H(s))$ has a band associated with the spectral projection $P(s)$ which is separated by a gap $g(s)$ from the rest. This setting leads to an adiabatic approximation where the error terms are $O\left(\frac{1}{\tau^{q}}\right)$ for some $q \geq 1$. There is a weaker form of the adiabatic theorem, where one does not require a spectral gap due to Avron-Elgart and Bornemann $\mathrm{AE}$, Bornemann]. In this case the estimate on the error term is $o(1)$ as $\tau$ goes to infinity.

There are several different viewpoints for a discussion of the quantum adiabatic theorem. Each one offers interesting insight. We shall only mention them briefly, since the main objective of this note is to give a short and concise proof of the adiabatic theorem in a setting which seems natural.

Berry [B] pointed out that time evolution of a quantum system in the adiabatic limit becomes geometric. Simon [SIM] noted that Berrys discovery can be interpreted as a parallel transport in a vector bundle with Berry's phase as the corresponding holonomy. Kato $[\mathrm{K}]$ used adiabatic time evolution as a powerful tool in his perturbation theory of linear operators. Born and Oppenheimer [BO] used the adiabatic approximation in order to separate fast and slow motion in molecules thereby explaining the qualitative picture of their spectra. Thouless and Niu NT, Avron and Seiler ASY1 showed that conductances in quantum Hall systems is defined in the adiabatic limit and is a topological number. 


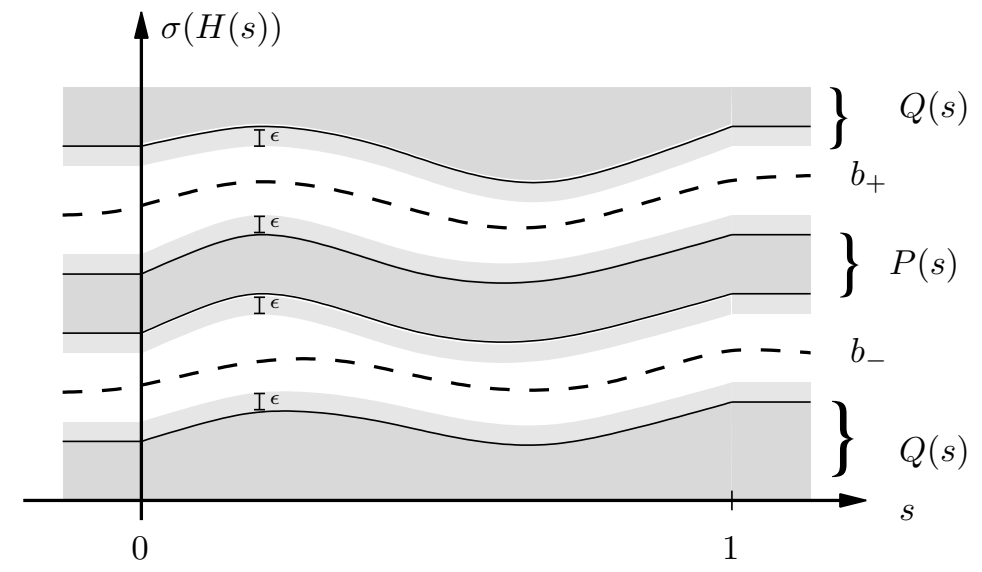

Figure 1: Energy band bordered by gaps. Shaded areas correspond to spectrum of $H(s)$. The projection $P(s)$ is associated with $\sigma(H(s)) \cap\left[b_{-}(s), b_{+}(s)\right]$, and $Q(s)=1-P(s)$.

\section{Statement of results}

In the following we will use the following notational conventions: The letter $P$ denotes an orthogonal projection and at the same time its range. $[A, B]$ is the commutator $A B-B A$ of the operators $A$ and $B$. $A^{*}$ is the adjoint of $A$. Furthermore we shall use regularly the following well known facts:

- Let $\Gamma$ be a positively oriented loop in the complex plane circling the spectrum associated with an orthogonal eigenprojection $P$ of a self-adjoint operator $H$. Then $P=-\frac{1}{2 \pi i} \oint_{\Gamma}(H-z)^{-1} d z$.

- Let $P(s)$ be a smooth family of orthogonal projections and $Q(s)=1-P(s)$ the projection on the orthogonal complement. Differentiating the relation $P^{2}(s)=P(s)$ gives

$$
\dot{P}(s)=P(s) \dot{P}(s) Q(s)+Q(s) \dot{P}(s) P(s) .
$$

An operator of this type is called "off-diagonal".

A summary of notation is given at the end of the paper (Table 11).

Let $I \subset \mathbb{R}$ be an interval with $0 \in I$ and $H(s), s \in I$, a family of Hamiltonians. We are interested in the time-dependent Schrödinger equations (11), where $\tau>0$ defines a time scale. A change of variables converts the family of equations (11) to the equivalent family (2). We will denote the unitary time evolution associated with 
(2) by $U_{\tau}(s)$.

Throughout the article, $H$ is assumed to fulfill the following conditions:

i. $H(s), s \in I$, are self-adjoint operators on a Hilbert space $\mathcal{H}$, with an $s$ independent domain $D$.

ii. $H(s)$ is a $k \geq 2$ times continuously differentiable map from $I$ to the space $B(D, \mathcal{H})$ of bounded linear operators from $D$ to $\mathcal{H}$ equipped with the graph norm of $H(0)$.

iii. $H(s)$ has gaps in the spectrum, and $P(s)$ is the spectral projection on a band bordered by gaps, i.e., there are two real-valued, continuous functions $b_{+}$and $b_{-}$, and $g>0$ such that

$$
\operatorname{dist}\left[\left\{b_{+}(s), b_{-}(s)\right\}, \sigma(H(s))\right]>g \quad(s \in I)
$$

and $P(s)$ is associated with the nonempty band $s \in I, \sigma(H(s)) \cap\left[b_{-}(s), b_{+}(s)\right]$.

Conditions $(i)$ and $(i i)$ imply that the resolvent $s \mapsto(H(s)-z)^{-1}$ as a map from $I$ to $B(\mathcal{H})$ is $k$ times continuously differentiable and ensure the existence and uniqueness of the propagator $U_{\tau}(s)$ if $k \geq 1$ (see e.g. [RS] p.285): $U_{\tau}(s)$ is unitary, strongly continuous in $s$, maps $D$ onto $D, U_{\tau}(0)=\mathrm{id}_{\mathcal{H}}$, and for each $\psi \in D, \psi_{\tau}(s):=U_{\tau}(s) \psi \in C^{1}(I, \mathcal{H})$ and satisfies (2). Condition (iii) is illustrated by Figure 1.

The first two adiabatic theorems give an estimate of the probability of finding a state $\psi$ initially in $P(0)$ outside $P(s)$ at time $s$ and compare $P_{\tau}(s):=U_{\tau}(s) P(0) U_{\tau}(s)^{*}$ to $P(s)$ for large time scales $\tau$. $P_{\tau}(s)$ is the time evolved projection, whereas $P(s)$ is the instantaneous spectral projection associated with the band of the spectrum of interest.

Theorem 1. (First adiabatic theorem.) Assuming the general conditions on $H$ stated above the following holds: For $\psi \in P(0)$ the transition probability is of order $O\left(1 / \tau^{2}\right)$ :

$$
\left(U_{\tau}(s) \psi,(1-P(s)) U_{\tau}(s) \psi\right)=O\left(1 / \tau^{2}\right)
$$

and

$$
\left\|P_{\tau}(s)-P(s)\right\|=O(1 / \tau)
$$

Both estimates hold uniformly on compact subsets of $I$. 
Theorem 2. (Second adiabatic theorem/switching theorem.) If in addition to the general conditions we assume that $\dot{H}$ is compactly supported in an interval $] 0,1[\subset I$ the above estimates can be improved to

$$
\begin{aligned}
\left(U_{\tau}(s) \psi,(1-P(s)) U_{\tau}(s) \psi\right) & =O\left(1 / \tau^{2(k-1)}\right) \\
\left\|P_{\tau}(s)-P(s)\right\| & =O\left(1 / \tau^{k-1}\right)
\end{aligned}
$$

uniformly in $I \backslash] 0,1[$.

Remarks concerning Theorem 2: If $\dot{H}$ is compactly supported in an interval $] \alpha, \omega[\subset I$ the previous estimates change into:

For $\psi \in P(\alpha)$

$$
\begin{aligned}
& \left(U_{\tau}(s ; \alpha) \psi,(1-P(s)) U_{\tau}(s ; \alpha) \psi\right)=O\left(1 / \tau^{2(k-1)}\right) \\
& \left\|U_{\tau}(s ; \alpha) P_{\tau}(s) U_{\tau}(s ; \alpha)^{*}-P(s)\right\|=O\left(1 / \tau^{k-1}\right),
\end{aligned}
$$

uniformly in $I \backslash] \alpha, \omega\left[. U_{\tau}(s ; \alpha)\right.$ denotes the propagator of the Schrödinger equation for the inital value $\alpha$.

For even more regular Hamiltonians, it is possible to get better bounds on the transition amplitudes using different methods of proof. For example, when the family of Hamiltonians $H(s)$ is in a Gevrey class, one can apply methods of phase space tunneling, see [J], or use Nencius technique [N2.

Theorem 3. Suppose the spectrum of $H(s)$ restricted to $P(s)$ consists of $m(s)$ eigenvalues (each possibly degenerate, crossing permitted) separated by a gap $g(s)$ from the rest of the spectrum of $H(s)$, and $H, \dot{H}$ and $\ddot{H}$ are bounded operators. (This assumption is always fulfilled in finite-dimensional spaces). Then for $\psi \in P(0)$ and any $s \in I$

$$
\left|\left(U_{\tau}(s) \psi,(1-P(s)) U_{\tau}(s) \psi\right)\right| \leq A(s)^{2}, \quad\left\|P_{\tau}(s)-P(s)\right\| \leq A(s)
$$

where

$$
\begin{aligned}
A(s) & =\left.\frac{1}{\tau} \frac{\sqrt{m}\|\dot{P}\|}{g}\right|_{u . b .}+\frac{1}{\tau} \int_{0}^{s}\left(\sqrt{m} \frac{\|Q \ddot{P} P\|+\|\dot{P}\|^{2}}{g}+2 m \frac{\|\dot{H}\|\|\dot{P}\|}{g^{2}}\right) \\
& \leq\left.\frac{1}{\tau} \frac{m\|\dot{H}\|}{g^{2}}\right|_{u . b .}+\frac{1}{\tau} \int_{0}^{s}\left(\frac{m\|\ddot{H}\|}{g^{2}}+7 m \sqrt{m} \frac{\|\dot{H}\|^{2}}{g^{3}}\right),
\end{aligned}
$$

and $\left.f\right|_{u . b .}$ is a shorthand for $f(0)+f(s)$.

Theorem 4. Suppose the assumptions of Theorem 4 are satisfied and $H$ is 3 times differentiable. Let $h(s):=\max \left(\|\dot{H}(s)\|,\|\ddot{H}(s)\|,\left\|\frac{d^{3}}{d s^{3}} H(s)\right\|\right)$. Then there is a constant $C$ (possibly depending on $m$ ) such that (6) holds with

$$
A(s)=\left.\frac{m h}{\tau g^{2}}\right|_{u . b .}+\frac{C}{\tau^{2}}\left(\left.\frac{h^{2}}{g^{4}}\right|_{u . b .}+\frac{h}{g^{2}}(0) \int_{0}^{s} \frac{h^{2}}{g^{3}}+\int_{0}^{s} \frac{h^{2}}{g^{5}}+\int_{0}^{s} d s^{\prime} \frac{h^{2}}{g^{3}}\left(s^{\prime}\right) \int_{0}^{s^{\prime}} d s^{\prime \prime} \frac{h^{2}}{g^{3}}\left(s^{\prime \prime}\right)\right) .
$$




\section{$3 \quad$ Notation and identities}

\subsection{Adiabatic Time Evolution and Wave Operator}

The proof of the adiabatic theorems is most easily accomplished with the aid of an idealized time evolution, the adiabatic time evolution introduced in ASY1, mapping the spectral subspace $P(0)$ onto $P(s)$. This is an adaptation of an idea by Kato to the present situation $[\mathrm{K}$.

¿From the gap condition $($ iii $)$ and $(H(\cdot)-z)^{-1} \in C^{k}(I, B(\mathcal{H}))$ it follows that $P(s)$ is in $C^{k}(I, B(\mathcal{H}))$ as well. Define the adiabatic Hamiltonian associated with $P$ and the time scale $\tau$ by

$$
H_{\tau}^{A}(s):=H(s)+\frac{i}{\tau}[\dot{P}(s), P(s)], \quad s \in I .
$$

$H_{\tau}^{A}$ satisfies the conditions (i) and (ii) with differentiability of order $k-1$. Existence of the corresponding time evolution $U_{\tau}^{A}(\cdot)$ is ensured for $k-1 \geq 1$.

The adiabatic time evolution generated by $\tau H_{\tau}^{A}(s)$ is ideal in the following sense:

Lemma 1. $U_{\tau}^{A}(\cdot)$ satisfies the intertwining property

$$
U_{\tau}^{A}(s) P(0)=P(s) U_{\tau}^{A}(s), \quad s \in I .
$$

Proof. The strategy of the proof is to show that both sides satisfy the same initial value problem $X(0)=P(0), \dot{X}(s)=-i \tau H_{\tau}^{A}(s) X(s)$ using that $\dot{P}$ is off-diagonal, see identity (5). (See also ASY1 p. 38.)

In the following we will omit the $s$-dependence and use the notation $Q=1-P, P_{0}$, $Q_{0}$ as a shorthand for $Q(s)=1-P(s), P(0)$ and $Q(0)$.

We prove the theorems by comparing the real time evolution $U_{\tau}(s)$ with the idealized time evolution $U_{\tau}^{A}(s)$. The wave operator $\Omega_{\tau}(s):=U_{\tau}^{A}(s)^{*} U_{\tau}(s)$ is a standard tool used in scattering theory to compare dynamics, here, $U_{\tau}$ and $U_{\tau}^{A}$. We expect that adiabatic and real time evolution are close, i.e., $\Omega_{\tau}$ is close to the identity, for big $\tau$. The proof of the adiabatic theorems reduces to the estimation of the off-diagonal blocks of the wave operator since for $\psi \in P_{0},\|\psi\|=1$

$$
\left(U_{\tau} \psi, Q U_{\tau} \psi\right)=\left\|Q U_{\tau} P_{0} \psi\right\|^{2}=\left\|Q U_{\tau}^{A} \Omega_{\tau} P_{0} \psi\right\|^{2} \leq\left\|Q_{0} \Omega_{\tau} P_{0}\right\|^{2}
$$

and

$$
\left\|P_{\tau}-P\right\|=\left\|U_{\tau} P_{0} U_{\tau}^{*}-U_{\tau}^{A} P_{0}\left(U_{\tau}^{A}\right)^{*}\right\|=\left\|\Omega_{\tau} P_{0}-P_{0} \Omega_{\tau}\right\|=\left\|Q_{0} \Omega_{\tau} P_{0}-P_{0} \Omega_{\tau} Q_{0}\right\| .
$$


By a straightforward calculation $\Omega_{\tau}$ satisfies the Volterra integral equation

$$
\Omega_{\tau}(s)=1-\int_{0}^{s} K_{\tau}\left(s^{\prime}\right) \Omega_{\tau}\left(s^{\prime}\right) d s^{\prime}
$$

where the kernel $K_{\tau}$ is defined by

$$
K_{\tau}(s):=U_{\tau}^{A}(s)^{*}[\dot{P}(s), P(s)] U_{\tau}^{A}(s)
$$

and the integral here and in the following is the Riemann integral in the strong sense.

\subsection{Integration by Parts Lemma}

With a map $X$ from $I$ to $B(\mathcal{H})$ we associate another map $X[\cdot]$ defined by

$$
X[s]:=U_{\tau}^{A}(s)^{*} X(s) U_{\tau}^{A}(s) .
$$

With this notation the integral kernel $K_{\tau}$ of the Volterra equation (10) introduced in the last section is just $[\dot{P}, P][\cdot]$ and the Volterra equation gives rise to integrals of the form $\int X[t] Y(t) d t$. Such integrals can be integrated by parts.

In the integral by parts formula (Lemma 3) the twiddle operation plays a crucial role. It can be understood as a partial inverse of the add operation $A \mapsto[H, A]$ as follows:

Lemma 2. (Twiddle operation.) Let $X$ be a map from $I$ to $B(\mathcal{H})$ and $\Gamma(s)$ a contour in $\mathbb{C}$ such that $P(s)=-\frac{1}{2 \pi i} \oint_{\Gamma(s)}(H(s)-z)^{-1} d z$. Define

$$
\tilde{X}(s):=\frac{1}{2 \pi i} \oint_{\Gamma(s)}(H(s)-z)^{-1} X(s)(H(s)-z)^{-1} d z .
$$

Then $P \tilde{X} P=Q \tilde{X} Q=0$ and on $D$

$$
\begin{gathered}
{[H, \tilde{X}]=P X-X P} \\
P\left[H_{\tau}^{A}, \tilde{X}\right] Q=P X Q, \quad Q\left[H_{\tau}^{A}, \tilde{X}\right] P=-Q X P .
\end{gathered}
$$

Proof. We drop the $s$-dependence. Let $\Gamma^{\prime}$ be a contour in $\mathbb{C}$ lying outside the domain delimited by $\Gamma$ and such that $P$ and $\tilde{X}$ can be written as integrals over $\Gamma^{\prime}$ instead of $\Gamma$. Then

$$
\begin{aligned}
\tilde{X} Q & =-\frac{1}{(2 \pi i)^{2}} \oint_{\Gamma}(H-z)^{-1} X(H-z)^{-1} \oint_{\Gamma^{\prime}}\left(\frac{1}{z-z^{\prime}}-\left(H-z^{\prime}\right)^{-1}\right) d z^{\prime} d z \\
& =-\frac{1}{(2 \pi i)^{2}} \oint_{\Gamma}(H-z)^{-1} X(H-z)^{-1} \oint_{\Gamma^{\prime}} \frac{1}{z-z^{\prime}}(H-z)^{-1}\left(H-z^{\prime}\right)^{-1} d z^{\prime} d z \\
& =-\frac{1}{(2 \pi i)^{2}} \oint_{\Gamma^{\prime}} \oint_{\Gamma} \frac{1}{z-z^{\prime}}(H-z)^{-1} d z X\left(H-z^{\prime}\right)^{-1} d z^{\prime} \\
& =\frac{1}{2 \pi i} \oint_{\Gamma^{\prime}} P\left(H-z^{\prime}\right)^{-1} X\left(H-z^{\prime}\right)^{-1} d z^{\prime}=P \tilde{X}
\end{aligned}
$$


This implies $P \tilde{X} P=0=Q \tilde{X} Q$. The commutator $[H, \tilde{X}]$ can be computed on $D \supset \mathcal{R}(\tilde{X})$ :

$$
\begin{aligned}
{[H, \tilde{X}] } & =\frac{1}{2 \pi i} \oint_{\Gamma}\left[H-z,(H-z)^{-1} X(H-z)^{-1}\right] d z \\
& =\frac{1}{2 \pi i} \oint_{\Gamma}\left(X(H-z)^{-1}-(H-z)^{-1} X\right) d z=-X P+P X .
\end{aligned}
$$

The second pair of identities follows with

$$
P[[\dot{P}, P], \tilde{X}] Q=(P[\dot{P}, P] P) \tilde{X} Q-P \tilde{X}(Q[\dot{P}, P] Q)=0,
$$

and similarly $Q[[\dot{P}, P], \tilde{X}] P=0$.

Lemma 3. (Partial integration.) Let $X \in C^{1}(I, B(\mathcal{H})), Y \in C^{0}(I, B(\mathcal{H}))$. Then $\tilde{X}$ defined as above is in $C^{1}(I, B(\mathcal{H}))$ and for $s \in I$

$\int_{0}^{s} P_{0} X\left[s^{\prime}\right] Q_{0} Y\left(s^{\prime}\right) d s^{\prime}=-\frac{i}{\tau}\left(\left.P_{0} \tilde{X}\left[s^{\prime}\right] Q_{0} Y\left(s^{\prime}\right)\right|_{0} ^{s}-\int_{0}^{s} P_{0} \dot{\tilde{X}}\left[s^{\prime}\right] Q_{0} Y\left(s^{\prime}\right) d s^{\prime}-\int_{0}^{s} P_{0} \tilde{X}\left[s^{\prime}\right] Q_{0} \dot{Y}\left(s^{\prime}\right) d s^{\prime}\right)$.

A similar equality holds with $P_{0}$ and $Q_{0}$ interchanged and an overall change of sign on the right-hand side.

Remark. The notation $X[s]$ is a shorthand for adjoining $\left(U_{\tau}^{A}\right)^{*}$ and $U_{\tau}^{A}$ after taking into account other symbols on $X$, e.g. $\dot{\tilde{X}}[s]=U_{\tau}^{A}(s)^{*} \dot{\tilde{X}}(s) U_{\tau}^{A}(s)$.

Proof. It suffices to notice that $\tilde{X}[\cdot]$ is strongly differentiable and

$$
\begin{aligned}
\frac{d}{d s} P_{0} \tilde{X}[s] Q_{0} & =P_{0}\left(i \tau\left[H_{\tau}^{A}, \tilde{X}\right][s]+\dot{\tilde{X}}[s]\right) Q_{0}=\left(i \tau P\left[H_{\tau}^{A}, \tilde{X}\right] Q\right)[s]+P_{0} \dot{\tilde{X}}[s] Q_{0} \\
& =P_{0}(i \tau X[s]+\dot{\tilde{X}}[s]) Q_{0},
\end{aligned}
$$

where the intertwining property has been used in the form $(P Z Q)[s]=P[s] Z[s] Q[s]=$ $P_{0} Z[s] Q_{0}$ for $Z: I \rightarrow B(\mathcal{H})$.

\section{Proof of Theorem 1 and Theorem 2}

Using the intertwining property, we see that the integral kernel of the Volterra equation (10) can be written as

$$
K_{\tau}(s)=(\dot{P} P-P \dot{P})[s]=(Q \dot{P} P-P \dot{P} Q)[s]=Q_{0} \dot{P}[s] P_{0}-P_{0} \dot{P}[s] Q_{0} .
$$


As a consequence, the Volterra equation (10) written for $Q_{0} \Omega_{\tau} P_{0}$ becomes

$$
Q_{0} \Omega_{\tau}(s) P_{0}=-\int_{0}^{s} Q_{0} \dot{P}\left[s^{\prime}\right] P_{0} \Omega_{\tau}\left(s^{\prime}\right) P_{0} d s^{\prime} .
$$

We can apply Lemma 3 and integrate by parts:

$$
\begin{gathered}
Q_{0} \Omega_{\tau}(s) P_{0}=-\frac{i}{\tau}\left(\left.Q_{0} \tilde{\dot{P}}\left[s^{\prime}\right] P_{0} \Omega_{\tau}\left(s^{\prime}\right) P_{0}\right|_{0} ^{s}-\int_{0}^{s} Q_{0} \dot{\dot{\dot{P}}}\left[s^{\prime}\right] P_{0} \Omega_{\tau}\left(s^{\prime}\right) P_{0} d s^{\prime}\right. \\
\left.-\int_{0}^{s} Q_{0} \tilde{\dot{P}}\left[s^{\prime}\right] P_{0} \dot{P}\left[s^{\prime}\right] Q_{0} \Omega_{\tau}\left(s^{\prime}\right) P_{0} d s^{\prime}\right) .
\end{gathered}
$$

Therefore $\left\|Q_{0} \Omega_{\tau} P_{0}\right\|=O(1 / \tau)$. The same method gives the bound $\left\|P_{0} \Omega_{\tau} Q_{0}\right\|=$ $O(1 / \tau)$, so that Theorem 1 follows with (8) and (9).

Now suppose $\dot{H}$ is compactly supported in ]0,1[. The Volterra equation (10) shows $\Omega_{\tau}(s)=1$ for $s \leq 0$ and $\Omega_{\tau}(s)=\Omega_{\tau}(1)$ for $s \geq 1$, hence it is enough to prove the statement for $s=1$. In equation (11), for $s \geq 1$, the boundary terms vanish. The basic idea to prove Theorem 2 is to estimate the two remaining integrals by iterating the integration by parts. But doing so naively for the second integral with $X(s)=$ $\dot{\dot{P}}(s)$ and $Y(s)=P[s] Q_{0} \Omega_{\tau}(s) P_{0}$ leads to derivatives of $P[s]=U_{\tau}^{A}(s)^{*} P(s) U_{\tau}^{A}(s)$ and thus to powers of $\tau$. This can be avoided by inserting the expression for $Q_{0} \Omega_{\tau} P_{0}$ recursively into the second integral. As a price to pay, we have to consider multiple integrals.

Lemma 4. Let $0 \leq m \leq k$. Then for all $j \in \mathbb{N}$ and $X_{1}, \ldots, X_{j} \in C_{0}^{m}(] 0,1[, B(\mathcal{H}))$ the integral

$$
A_{j}\left(X_{1}, \ldots, X_{j}\right)=Q_{0} \int_{0}^{1} d s_{1} X_{1}\left[s_{1}\right] Q_{0} \ldots Q_{0} \int_{0}^{s_{j-1}} d s_{j} X_{j}\left[s_{j}\right] P_{0} \Omega_{\tau}\left(s_{j}\right) P_{0}
$$

is of order $O\left(1 / \tau^{m}\right)$.

Remark. In this notation, $Q_{0} \Omega_{\tau}(1) P_{0}=-A_{1}(\dot{P})$.

Proof. By induction over $m$. The statement for $m=0$ is obvious since all $A_{j}$ are bounded as functions of $\tau$. For the induction step $m \leq k-1 \rightarrow m+1$ assume $X_{1}, . ., X_{j}$ are in $C_{0}^{m+1}(] 0,1[, B(\mathcal{H}))$. Suppose first $j \geq 2$. We integrate by parts with respect to the variable $s_{j}$ and use the vanishing of boundary terms:

$$
\begin{aligned}
& \int_{0}^{s_{j-1}} Q_{0} X_{j}\left[s_{j}\right] P_{0} \Omega_{\tau}\left(s_{j}\right) P_{0} d s_{j}=\frac{i}{\tau}\left(Q_{0} \tilde{X}_{j}\left[s_{j-1}\right] P_{0} \Omega_{\tau}\left(s_{j-1}\right) P_{0}\right. \\
& \left.\quad-\int_{0}^{s_{j-1}} Q_{0} \dot{\tilde{X}}_{j}\left[s_{j}\right] P_{0} \Omega_{\tau}\left(s_{j}\right) P_{0} d s_{j}-\int_{0}^{s_{j-1}} Q_{0} \tilde{X}_{j}\left[s_{j}\right] P_{0} \dot{P}\left[s_{j}\right] Q_{0} \Omega_{\tau}\left(s_{j}\right) P_{0} d s_{j}\right) \\
& =\frac{i}{\tau}\left(Q_{0} \tilde{X}_{j}\left[s_{j-1}\right] P_{0} \Omega_{\tau}\left(s_{j-1}\right) P_{0}-\int_{0}^{s_{j-1}} Q_{0} \dot{\tilde{X}}_{j}\left[s_{j}\right] P_{0} \Omega_{\tau}\left(s_{j}\right) P_{0} d s_{j}\right. \\
& \left.\quad+\int_{0}^{s_{j-1}} d s_{j} Q_{0}\left(\tilde{X}_{j} P \dot{P}\right)\left[s_{j}\right] Q_{0} \int_{0}^{s_{j}} d s_{j+1} \dot{P}\left[s_{j+1}\right] P_{0} \Omega_{\tau}\left(s_{j+1}\right) P_{0}\right) .
\end{aligned}
$$


Inserting this into the definition of $A_{j}$, we get

$$
\begin{gathered}
A_{j}\left(X_{1}, . ., X_{j}\right)=\frac{i}{\tau}\left(A_{j-1}\left(X_{1}, . . X_{j-2}, X_{j-1} Q \tilde{X}_{j}\right)-A_{j}\left(X_{1}, . ., X_{j-1}, \dot{\tilde{X}}_{j}\right)\right. \\
\left.-A_{j+1}\left(X_{1}, . . X_{j-1}, \tilde{X}_{j} P \dot{P}, \dot{P}\right)\right) .
\end{gathered}
$$

This remains valid for $j=1$ if we set $A_{0}:=0$. The new variables $X_{j-1} Q \tilde{X}_{j}, \dot{\tilde{X}}_{j}, \tilde{X}_{j} P \dot{P}, \dot{P}$ are in $C_{0}^{m}(] 0,1[, B(\mathcal{H}))$ (here we use $\left.m \leq k-1\right)$. Hence

$$
A_{j}\left(X_{1}, . ., X_{j}\right)=\frac{i}{\tau} O\left(\frac{1}{\tau^{m}}\right)=O\left(\frac{1}{\tau^{m+1}}\right) .
$$

Proof of Theorem 2. Suppose $\dot{H}$ is compactly supported in ]0,1[. Then $\dot{P}$ belongs to $C_{0}^{k-1}(] 0,1[, B(\mathcal{H}))$. Lemma 4 implies $Q_{0} \Omega_{\tau}(1) P_{0}=-A_{1}(\dot{P})=O\left(1 / \tau^{k-1}\right)$. The lemma still holds with $Q$ and $P$ interchanged so $P_{0} \Omega_{\tau}(1) Q_{0}=O\left(1 / \tau^{k-1}\right)$. Together with (8) and (9) this concludes the proof.

\section{Dependence on the gap}

Up to now, we have examined the dependence of the error terms on the time scale $\tau$ only. In order to give error bounds with explicit gap dependence, we need to know how $P$ decorated with dots and tildas behaves. The aim of the following series of lemmas is to generalize the bounds given in $[\mathrm{R}$, pp. $8-9$ to the case where $P$ projects on more than one eigenvalue. These allow to prove Theorem 3 and 4 . The reader interested in projections $P(s)$ associated with a single (possibly degenerate) eigenvalue can find a simpler proof of the inequalities stated in Lemma 8 , up to a constant factor, in $[\mathrm{R}$.

We will assume throughout this section that all operators are bounded and that PHP has discrete spectrum. This is always fulfilled in finite-dimensional spaces. We write

$$
P(s)=\sum_{j=1}^{m(s)} P_{j}(s), \quad H(s) P_{j}(s)=\lambda_{j}(s) P_{j}(s),
$$

where $P_{j}(s)$ are the projections associated with the $m(s)$ eigenvalues $\lambda_{1}(s), . ., \lambda_{m(s)}(s)$. Note that each eigenvalue might be degenerate and the eigenvalues are allowed to cross. Furthermore, let

$$
g(s):=\operatorname{dist}\left(\left\{\lambda_{1}(s), . ., \lambda_{m(s)}\right\}, \sigma(H(s)) \backslash\left\{\lambda_{1}(s), . ., \lambda_{m(s)}(s)\right\}\right)
$$

denote the gap in $s$. In the following we will drop the $s$-dependence and use the notation

$$
R_{z}=(H(s)-z)^{-1}, \quad \hat{R}_{z}=Q\left[\left.(H(s)-z)\right|_{Q}\right]^{-1} Q
$$


Note that the reduced resolvent $\hat{R}_{z}$ is well-defined even if $z$ is one of the eigenvalues $\lambda_{j}$, and $\left\|\hat{R}_{\lambda_{j}}\right\| \leq 1 / g$.

Lemma 5. Let $X, A, B$ be bounded operators. Then

$$
\begin{gathered}
\tilde{X}=-\sum_{j=1}^{m}\left(P_{j} X \hat{R}_{\lambda_{j}}+\hat{R}_{\lambda_{j}} X P_{j}\right) \\
\frac{1}{2 \pi i} \oint_{\Gamma} R_{z} A R_{z} B R_{z} d z=(P-Q)(\tilde{A} \tilde{B}+\widetilde{A \tilde{B}}-\widetilde{\tilde{A} B})
\end{gathered}
$$

Proof. By Lemma 2, $[H, X]=[P, X]$. This implies

$$
P_{j}[H, X] Q=\lambda_{j} P_{j} X Q-P_{j} X Q H Q=P_{j} X Q
$$

hence

$$
P X Q=\sum_{j} P_{j} X Q=-\sum_{j} P_{j} X \hat{R}_{\lambda_{j}}
$$

Similarly, $Q X P=-\sum_{j} \hat{R}_{\lambda_{j}} X P_{j}$. Since by Lemma $2 \tilde{X}$ is off-diagonal, equation (12) follows. Now let

$$
G(A, B):=\frac{1}{2 \pi i} \oint_{\Gamma} R_{z} A R_{z} B R_{z} d z
$$

Then

$$
[H, G(A, B)]=\frac{1}{2 \pi i} \oint_{\Gamma}\left[H-z, R_{z} A R_{z} B R_{z}\right] d z=A \tilde{B}-\tilde{A} B
$$

An argument similar to the reasoning bevor fixes the off-diagonal part of $G(A, B)$ :

$$
P G(A, B) Q=P(\widetilde{A \tilde{B}}-\widetilde{\tilde{A} B}) Q, \quad Q G(A, B) P=-Q(\widetilde{A \tilde{B}}-\widetilde{\tilde{A} B}) P .
$$

The diagonal blocks of $G(A, B)$ can be computed, using that $z \mapsto \hat{R}_{z}$ is holomorphic in the domain enclosed by $\Gamma$ and has $\hat{R}_{z}^{2}$ as its derivative with respect to $z$.

$$
\begin{aligned}
Q G(A, B) Q & =\sum_{j} \frac{1}{2 \pi i} \oint_{\Gamma} \frac{1}{\lambda_{j}-z} \hat{R}_{z} A P_{j} B \hat{R}_{z} d z+\frac{1}{2 \pi i} \oint_{\Gamma} \hat{R}_{z} A \hat{R}_{z} B \hat{R}_{z} d z \\
& =-\sum_{j} \hat{R}_{\lambda_{j}} A P_{j} B \hat{R}_{\lambda_{j}}+0=-Q \tilde{A} \tilde{B} Q .
\end{aligned}
$$

Similarly,

$$
\begin{aligned}
P G(A, B) P= & \sum_{j, k} \frac{1}{2 \pi i} \oint_{\Gamma} \frac{1}{\lambda_{j}-z} \frac{1}{\lambda_{k}-z} P_{j} A \hat{R}_{z} B P_{k} d z \\
& +\sum_{j, k, l} \frac{1}{2 \pi i} \oint_{\Gamma} \frac{1}{\left(\lambda_{j}-z\right)\left(\lambda_{k}-z\right)\left(\lambda_{l}-z\right)} d z P_{j} A P_{k} B P_{l} .
\end{aligned}
$$


But now

$$
\frac{1}{2 \pi i} \oint_{\Gamma} \frac{1}{\left(\lambda_{j}-z\right)^{2}} \hat{R}_{z}=R_{\lambda_{j}}^{2}
$$

for $j \neq k$

$$
\frac{1}{2 \pi i} \oint_{\Gamma} \frac{1}{\left(\lambda_{j}-z\right)\left(\lambda_{k}-z\right)} R_{z}=\frac{1}{\lambda_{j}-\lambda_{k}}\left(\hat{R}_{\lambda_{j}}-\hat{R}_{\lambda_{k}}\right)=\hat{R}_{\lambda_{j}} \hat{R}_{\lambda_{k}}
$$

and $\oint_{\Gamma}\left[\left(\lambda_{j}-z\right)\left(\lambda_{k}-z\right)\left(\lambda_{l}-z\right)\right]^{-1} d z=0$ for any $j, k, l \in\{1, . ., m(s)\}$, so that

$$
P G(A, B) P=\sum_{j, k} P_{j} A \hat{R}_{\lambda_{j}} \hat{R}_{\lambda_{k}} B P_{k}=P \tilde{A} \tilde{B} P
$$

Putting together (14), (15) and (16) and making use of the off-diagonal character of any "twiddled" operator $\tilde{A}, \tilde{B}$ etc. gives (13).

Lemma 6. We have $\dot{P}=\tilde{\dot{H}}$. Let $X$ be a continuously differentiable map from $I$ to $B(\mathcal{H})$. Then

$$
\dot{\tilde{X}}=\tilde{\dot{X}}+(Q-P)(\dot{P} \tilde{X}+\tilde{X} \dot{P}+\widetilde{[\dot{H}, \tilde{X}}]-\widetilde{[\dot{P}, X]})
$$

In particular,

$$
Q \dot{\tilde{\dot{P}}} P=Q(\tilde{\ddot{P}}+\widetilde{\dot{H} \tilde{\dot{P}}}-\widetilde{\tilde{\dot{P}} \dot{H}}) P \text {. }
$$

Proof. First notice

$$
\dot{P}=-\frac{d}{d s} \frac{1}{2 \pi i} \oint_{\Gamma} R_{z} d z=\frac{1}{2 \pi i} \oint R_{z} \dot{H} R_{z} d z=\dot{\dot{H}}
$$

Identity (17) then follows with

$$
\dot{\tilde{X}}=\frac{d}{d s} \frac{1}{2 \pi i} \oint_{\Gamma} R_{z} X R_{z} d z=\frac{1}{2 \pi i} \oint_{\Gamma}\left(R_{z} \dot{X} R_{z}-R_{z} \dot{H} R_{z} X R_{z}-R_{z} X R_{z} \dot{H} R_{z}\right) d z
$$

and Lemma 5. Since $\dot{P} \tilde{X}$ and $\tilde{X} \dot{P}$ are diagonal operators and $[\dot{P}, \dot{P}]=0$, (18) follows from (17) applied to $X=\dot{P}$.

Lemma 7. Let $X:[0,1] \rightarrow B(\mathcal{H})$. Then

$$
\|\tilde{X}(s)\| \leq \sqrt{m(s)} \frac{\|X(s)\|}{g(s)} .
$$

Proof. Let $\phi \in \mathcal{H}$.

$$
\begin{aligned}
\|\tilde{X} \phi\|=\|(P \tilde{X} Q+Q \tilde{X} P) \phi\|^{2} & =\|P \tilde{X} Q \phi\|^{2}+\|Q \tilde{X} P \phi\|^{2} \\
& \leq \max \left(\|P \tilde{X} Q\|^{2},\|Q \tilde{X} P\|^{2}\right)\|\phi\|^{2} .
\end{aligned}
$$


Moreover,

$$
Q \tilde{X} P=-\sum_{j=1}^{m} \hat{R}_{\lambda_{j}} X P_{j}=\left(P \tilde{X}^{*} Q\right)^{*}
$$

and for bounded operators $\|A\|=\left\|A^{*}\right\|$, hence it is enough to prove

$$
\|P X Q\| \leq \sqrt{m} \frac{\|X\|}{g}
$$

But this follows from $\|P X Q\|=\left\|(P X Q)^{*}(P X Q)\right\|^{1 / 2}$ and

$$
\begin{aligned}
\|P X Q\|^{2} & =\left\|(P X Q)^{*}(P X Q)\right\|=\left\|\sum_{j=1}^{m} \hat{R}_{\lambda_{j}} X^{*} P_{j} X \hat{R}_{\lambda_{j}}\right\| \\
& \leq m \frac{1}{g^{2}}\|X\|\left\|X^{*}\right\|=m \frac{\|X\|^{2}}{g^{2}}
\end{aligned}
$$

Lemma 8. The following series of bounds holds:

$$
\begin{aligned}
\|\dot{P}\| & \leq \sqrt{m} \frac{\|\dot{H}\|}{g} \\
\|\tilde{\dot{P}}\| & \leq \sqrt{m} \frac{\|\dot{P}\|}{g} \leq m \frac{\|\dot{H}\|}{g^{2}} \\
\|Q \ddot{P} P\| & \leq \sqrt{m} \frac{\|\ddot{H}\|}{g}+\frac{4 m\|\dot{H}\|^{2}}{g^{2}} \\
\|Q \dot{\tilde{P}} P\| & \leq \sqrt{m} \frac{\|Q \ddot{P} P\|}{g}+\frac{2 m\|\dot{H}\|\|\dot{P}\|}{g^{2}} \leq \frac{m\|\ddot{H}\|}{g^{2}}+6 \frac{m \sqrt{m}\|\dot{H}\|^{2}}{g^{3}} .
\end{aligned}
$$

More generally, let $\Gamma X:=\tilde{X}, D X:=\dot{X}$, then if $H$ is $(l+1)$-times differentiable and $h(s):=\max _{1 \leq j \leq l+1}\left\|\left(\frac{d}{d s}\right)^{j} H(s)\right\|$, there exists a constant $C_{l}$, possibly depending on the number $m$ of eigenvalues, such that

$$
\left\|(D \Gamma)^{l} D P\right\| \leq \frac{C_{l}}{g^{l}} \sup _{\alpha_{1}+. .+\alpha_{k}=l+1, \alpha_{j} \geq 1} \prod_{1 \leq j \leq k} \frac{1}{g}\left\|\frac{d^{\alpha_{j}} H}{d s^{\alpha_{j}}}\right\| \leq \frac{C_{l}}{g^{l}}\left(\frac{h}{g}\right)^{l+1},
$$

that is, every tilda pulls out a $1 / \mathrm{g}$ and every dot a $\mathrm{h} / \mathrm{g}$.

Proof. The proof of the series of bounds is straightforward with Lemma 6 and 8 , The estimate (20) is shown with an induction over $l$.

Theorem 3 now is a simple consequence of (11) and Lemma 7 If $H$ is 3 times 
differentiable, iterating the integration by parts gives the expansion

$$
\begin{aligned}
& Q_{0} \Omega_{\tau}(s) P_{0}=\left.\frac{i}{\tau} Q_{0} \tilde{\dot{P}}\left[s^{\prime}\right] \Omega_{\tau}\left(s^{\prime}\right) P_{0}\right|_{0} ^{s}+\left.\frac{1}{\tau^{2}} Q_{0} \dot{\tilde{\dot{P}}}\left[s^{\prime}\right] \Omega_{\tau}\left(s^{\prime}\right) P_{0}\right|_{0} ^{s} \\
& \left.\quad-\frac{1}{\tau^{2}} \int_{0}^{s} Q_{0}(\tilde{\dot{P}} \dot{P})\left[s^{\prime}\right] d s^{\prime}\right) \tilde{\dot{P}}[0] P_{0}-\frac{1}{\tau^{2}} \int_{0}^{s} Q_{0}(\dot{\tilde{\dot{P}}} P+\stackrel{\tilde{\dot{P}}}{\dot{P}}-\tilde{\dot{P}} \dot{P} \dot{\dot{P}})\left[s^{\prime}\right] \Omega_{\tau}\left(s^{\prime}\right) P_{0} d s^{\prime} \\
& \quad+\frac{1}{\tau^{2}} \int_{0}^{s} Q_{0}(\tilde{\dot{P}} \dot{P})\left[s^{\prime}\right] \int_{0}^{s^{\prime}}(\dot{\tilde{\dot{P}}} P+\dot{\dot{P}} \dot{P})\left[s^{\prime \prime}\right] \Omega_{\tau}\left(s^{\prime \prime}\right) P_{0} d s^{\prime \prime} d s^{\prime}
\end{aligned}
$$

Theorem 4 follows from this expansion and Lemma 8 ,

Comment on the traditional "adiabatic criterion". How big must $\tau$ be in order to ensure that the error $\left\|Q_{0} \Omega_{\tau} P_{0}\right\|$ remains smaller than some constant $\epsilon<1$ ? A frequently given answer $(\underline{M})$ is

$$
\tau \geq \mathrm{const} \sup _{0 \leq s \leq 1} \frac{\|\dot{H}(s)\|}{g(s)^{2}} .
$$

This criterion comes from the first order term in the expansion (21) which we rewrite

$$
\left\|Q_{0} \Omega_{\tau}(s) P_{0}\right\| \leq \frac{1}{\tau}\left(\frac{m\|\dot{H}\|}{g^{2}}(0)+\frac{m\|\dot{H}\|}{g}(s)\right)+\frac{C(H)}{\tau^{2}} .
$$

However, $C(H)$ in the second order term may depend on the gap. For a fixed Hamiltonian, one can always justify neglecting higher order times by taking $\tau$ sufficiently large. However, in applications to quantum computation one is often interested in a family of Hamiltonians in which the gap may change. Indeed, in the situations considered in Theorems 3 and 4, the second order coefficient $C(H)$ can be $O\left(1 / g^{6}\right)$. In such a case this term is unbounded for vanishing $g$ unless $\tau=O\left(1 / g^{3}\right)$.

\section{Applications to interpolating Hamiltonians}

In adiabatic quantum computation, the Hamiltonians of interest are interpolations between an initial Hamiltonian $H_{0}$ with an easily computable ground state and a final Hamiltonian $H_{1}$ whose ground state encodes the solution to some problem. The running time of an "adiabatic algorithm" is closely related to the time $\tau$ required to ensure a good agreement between real and adiabatic time evolution and depends crucially on the minimal gap $g_{\text {min }}$.

At first glance, Theorem [3] does not imply the general wisdom that $\tau$ should be of order $1 / g_{\min }^{2}$ : if we estimate the integrals by the maximum of the integrand, we get a worst case dependence in $1 / g_{\text {min }}^{3}$. A closer look at the following example however shows that it is not a good idea to bound the integral in this way and that additional 
knowledge about the gap function $g(s)$ does allow to extract the $1 / g_{\min }^{2}$ behavior from Theorem 3. Moreover, as suggested in VMZ, RC, it is possible to improve the error dependence on the minimal gap by adapting the interpolation between $H_{0}$ and $H_{1}$.

Example. (See also VMZ].) Let $|0\rangle,|1\rangle$ denote a basis of $\mathbb{C}^{2}$. For $w \in\{0,1\}^{n}$, let $|w\rangle=\left|w_{1}\right\rangle \otimes \ldots \otimes\left|w_{n}\right\rangle, \mathcal{H}:=\left(\mathbb{C}^{2}\right)^{\otimes n}$,

$$
H_{0}:=1-|\hat{0}\rangle\left\langle\hat{0}\left|, \quad H_{1}:=1-\right| u\right\rangle\langle u|,
$$

where $|\hat{0}\rangle=2^{-n / 2} \sum_{w \in\{0,1\}^{n}}|w\rangle$ and $u \in\{0,1\}^{n}$. $u$ is regarded as an unknown element to be searched for in a list of length $2^{n}$. We claim that:

1. Let $H(s)=(1-s) H_{0}+s H_{1}$. Then $g_{\text {min }}=2^{-n / 2}$, and there is a constant $C>0$ such that $\left\|Q_{0} \Omega_{\tau}(1) P_{0}\right\| \leq C /\left(\tau g_{\text {min }}^{2}\right)$.

2. Let $H(s)=(1-f(s)) H_{0}+f(s) H_{1}$. It is possible to choose $f:[0,1] \rightarrow[0,1]$ onto, monotone strictly increasing, such that $\left\|Q_{0} \Omega_{\tau}(1) P_{0}\right\| \leq C /\left(\tau g_{\text {min }}\right)$ for some constant $C>0$.

Proof. $H(s)=(1-s) H_{0}+s H_{1}$ has a non-degenerate ground state separated from the first excited state by the gap

$$
g(s)=\sqrt{2^{-n}+4\left(1-2^{-n}\right)\left(s-\frac{1}{2}\right)^{2}} .
$$

It is 1 at the boundaries $s=0,1$. We notice that for $p>1, \int_{0}^{1} g^{-p}(s) d s=O\left(g_{\text {min }}^{1-p}\right)$ and $\|\dot{H}\|=\left\|H_{1}-H_{0}\right\| \leq 2$. The first claim now follows from Theorem 3 .

Next, $H(s)=(1-f(s)) H_{0}+f(s) H_{1}$ has gap $g(f(s))$ with $g$ defined in (22). Let $f:[0,1] \rightarrow[0,1]$ be the solution of

$$
f(0)=0, \quad \dot{f}(s)=k g^{p}(f(s)), \quad k=\int_{0}^{1} g^{-p}(u) d u .
$$

Then $f(1)=1$. If $p>1, k=O\left(g_{\text {min }}^{1-p}\right)$. Since

$$
\dot{H}(s)=k g^{p}(f(s))\left(H_{1}-H_{0}\right), \quad \ddot{H}(s)=k^{2} g^{2 p-1}(f(s)) \dot{g}(f(s))\left(H_{1}-H_{0}\right),
$$

Theorem 3 gives

$$
\begin{aligned}
\left\|Q_{0} \Omega_{\tau}(s) P_{0}\right\| & \leq \frac{1}{\tau}\left\{4 k+k^{2} \int_{0}^{1}\left[2 g^{2 p-3}(f(s)) \mid \dot{g}\left(f(s) \mid+28 g^{2 p-3}(f(s)] d s\right\}\right.\right. \\
& =\leq \frac{k}{\tau}\left\{4+\int_{0}^{1}\left[2 g^{p-3}(u)|\dot{g}(u)|+28 g^{p-3}(u)\right] d u\right\}
\end{aligned}
$$


(we used the change of variables $u=f(s)$ ). For $p<2, \int g^{p-3}=O\left(g_{\min }^{p-2}\right) . g$ is decreasing on $[0,1 / 2]$, increasing on $[1 / 2,1]$, therefore for $p<2$

$$
\int_{0}^{1} g^{p-3}|\dot{g}|=\frac{2}{p-2}\left(1-g_{\min }^{p-2}\right)=O\left(g_{\min }^{p-2}\right) .
$$

Hence for $1<p<2\left\|Q_{0} \Omega_{\tau}(1) P_{0}\right\|=O\left(\tau^{-1} g_{\min }^{1-p} g_{\min }^{p-2}\right)=O\left(\left(\tau g_{\min }\right)^{-1}\right)$. This concludes the proof.

More generally, the previous considerations concerning the gap dependence will apply provided the gap function $g(s)$ has the three following properties:

- The gap at the boundaries $(s=0, s=1)$ is of order 1 .

- The gap decreases strictly towards its minimal value $g_{\min }$ and then increases strictly.

- If $p>1$, then $\int_{0}^{1} g^{-p}(s) d s=O\left(g_{\min }^{1-p}\right)$.

The first two features are shared by most examples studied so far (see e.g. [R, [SMS]). The third one needs to be checked more carefully, but it is reasonable to expect that $\int g^{-p}$ behaves better than $g_{\min }^{-p}$.

\begin{tabular}{|c|c|}
\hline$U_{\tau}(s)$ & real time evolution \\
\hline$U_{\tau}^{A}(s)$ & adiabatic time evolution \\
\hline$X[s]$ & $U_{\tau}^{A}(s)^{*} X(s) U_{\tau}^{A}(s)$ \\
\hline$\tilde{X}(s)$ & $\frac{1}{2 \pi i} \oint_{\Gamma(s)}(H(s)-z)^{-1} X(H(s)-z)^{-1} d z$ \\
\hline$\Omega_{\tau}(s)$ & $U_{\tau}^{A}(s)^{*} U_{\tau}(s) \quad$ wave operator \\
\hline$R_{z}$ & $(H(s)-z)^{-1} \quad$ resolvent \\
\hline$\hat{R}_{z}$ & $Q\left[\left.(H(s)-z)\right|_{Q}\right]^{-1} Q$ \\
\hline
\end{tabular}

Table 1: Summary of notation

\section{References}

[AR] A. Ambainis, O. Regev: An Elementary Proof of the Quantum Adiabatic Theorem. quant-ph/0411152 
[AE] J. E. Avron, A. Elgart: Adiabatic Theorem without a Gap Condition. Commun. Math. Phys. 203, 445-463 (1999)

[Bornemann] V. Bornemann: Homogenization in Time of Singular Perturbed Mechanical Systems Lecture Notes in Mathematics, Vol. 1687 (1998)

[ASY1] J. E. Avron, R. Seiler, L. G.Yaffe: Adiabatic Theorems and Applications to the Quantum Hall Effect. Commun. Math. Phys. 110, 33-49 (1987)

[ASY2] J. E. Avron, R. Seiler, L. G. Yaffe: Adiabatic Theorems and Applications to the Quantum Hall Effect: Erratum. Commun. Math. Phys. 156, 649-650 (1993)

[B] M. V. Berry: Quantal phase factors accompanying adiabatic changes. Proc. R. Soc. Lond. A 392, 45-47 (1964)

[BF] M. Born, V. Fock:Beweis des Adiabatensatzes. Z. Physik. 51, 165-169 (1928)

[BO] M. Born, R. Oppenheimer: Zur Quantentheorie der Molekeln. Ann. Phys. 84, 457-484 (1927)

[FGGS] E. Farhi, J. Goldstone, S. Gutmann, M. Spiser: Quantum Computation by Adiabatic Evolution. quant-ph/0001106

[FGG1] E. Farhi, J. Goldstone, G. Sam, J. Lapan, A. Lundgren, D. Preda: A Quantum Adiabatic Evolution Algorithm Applied to Random Instances of an NP-complete Problem. Science 292, 472-474 (20 April 2001); see also quant-ph/0104129

[FGG2] E. Farhi, J. Goldstone, S. Gutmann: Quantum Adiabatic Evolution Algorithms with Different Paths. quant-ph/0208135

[HJ] G. A. Hagedorn, A. Joye: Elementary exponential error estimates for the adiabatic approximation. J. Math. Anal. Appl. 267, 235-246 (2002)

[J] K. Jung: Adiabatic Theorem for Switching Processes with Gevrey Class Regularity. Technical University of Berlin Sfb 288 Preprint No. 442 and Dissertation, 1997

[JP] A. Joye, C. Pfister: Full asymptotic expansion of transition probabilities in the adiabatic limit. J. Phys. A 24, 753-766 (1991)

[K] T. Kato: On the adiabatic theorem of quantum mechanics. J.Phys. Soc. Japan $5,435-439$ (1950)

[KS] M. Klein, R. Seiler: Power-Law Corrections to the Kubo Formula Vanish in Quantum Hall Systems. Commun. Math. Phys. 128, 141-160 (1990).

[LL] L. Landau L. Lifshitz, Quantum Mechanics. Second Edition of English translation, Pergamon Press, 1965. 
[MEP] R. MacKenzie, E. Marcotte, H. Paquette: A new perturbative approach to the adiabatic approximation. quant-ph/0510024

[MS] K-P. Marzlin, B.C Sanders: Inconsistency in the application of the adiabatic theorem. Phys. Rev. Lett. 93, 160408-160411 (2004)

[M] A. Messiah: Quantum Mechanics, Vol. II. North-Holland Publishing Company (1962)

[N1] G. Nenciu: On the adiabatic theorem of quantum mechanics. J. Phys. A 13, L15-L18 (1980)

[N2] G. Nenciu: Linear Adiabatic Theory. Exponential Estimates. Commun. Math. Phys. 152, 479-496 (1993)

[NT] Q. Niu, D. J. Thouless: Quantised adiabatic charge transport in the presence of substrate disorder and many body interactions. J. Phys. A 17 (30-49)

[R] B. Reichardt: The quantum adiabatic optimization algorithm and local minima. STOC 2004

[RC] J. Roland, N. Cerf: Quantum search by local adiabatic evolution. Phys. Rev. A 65, 042308 (2002), quant-ph/0107015

[SMS] G. Schaller, S. Mostame, R. Schützhold: A Note on Adiabatic Quantum Computing. quant-ph/0510183

[SIM] B. Simon: Holonomy, the Quantum Adiabatic Theorem, and Berry's Phase Phys. Rev. Lett. 51, 2167-2170 (1983)

[TSKO] D. M. Tong, K. Singh, L. C. Kwek, C. H. Oh: Quantitative conditions do not guarantee the validity of the adiabatic approximation. Phys. Rev. Lett. 95, 110407 (2005); quant-ph/0509073

[VMZ] W. van Dam, M. Mosca, U. Vazirani: How powerful is adiabatic quantum computation? 42nd IEEE Symposium on Foundations of Computer Science (Las Vegas, NV, 2001), 279-287; quant-ph/0206003

[RS] M. Reed, B. Simon: Methods of Modern Mathematical Physics, Vol. II: Fourier Analysis, Self-Adjointness. Academic Press (1975) 